\title{
GuideLoc: UAV-Assisted Multitarget Localization System for Disaster Rescue
}

\author{
Anwen Wang, ${ }^{1}$ Xiang Ji, ${ }^{1}$ Dajun Wu, ${ }^{2}$ Xuedong Bai, ${ }^{1}$ Nana Ding, ${ }^{1}$ Jing Pang, \\ Shaofeng Chen, ${ }^{1}$ Xiaojiang Chen, ${ }^{1}$ and Dingyi Fang ${ }^{1}$ \\ ${ }^{1}$ School of Information Science and Technology, Northwest University, Xian, China \\ ${ }^{2}$ Institute for Infocomm Research, Singapore \\ Correspondence should be addressed to Dajun Wu; djwu@i2r.a-star.edu.sg and Dingyi Fang; dyf@nwu.edu.cn
}

Received 3 October 2016; Accepted 10 January 2017; Published 16 March 2017

Academic Editor: George Ghinea

Copyright (C) 2017 Anwen Wang et al. This is an open access article distributed under the Creative Commons Attribution License, which permits unrestricted use, distribution, and reproduction in any medium, provided the original work is properly cited.

\begin{abstract}
Locating trapped targets using the signals of wireless devices such as mobile phones carried by targets is increasingly becoming the preferred scheme for disaster rescue. However, the accuracy of radio-localization technology currently adopted in the rescue is not high enough. To solve such problems, researchers proposed the approaches of Warwalking or Wardriving. However, both approaches are all limited by application scenarios. This paper proposes GuideLoc, a highly efficient aerial wireless localization system, which uses the target guiding technology based on region division. GuideLoc allows an unmanned aerial vehicle (UAV) to fly over a target device and provide position coordinates of the UAV as the target location information. During the process of positioning targets, based on the result of regional division, GuideLoc uses a number of fixed antennas to get the received signal strength indicator (RSSI) and angle of arrival (AOA) information of the target and estimates the target location using the information and correspondingly controls the UAV to fly towards the target. Averaging method is also applied for determining coordinates of the target. Experiment results show that GuideLoc can in short time achieve 2.7-meter positioning accuracy in average. Compared with other wireless localization methods using UAVs proposed in the literature, it shortened the flight distance by more than $50 \%$. In addition, energy efficiency is also improved significantly.
\end{abstract}

\section{Introduction}

How many people died due to lack of timely rescue after an earthquake? Obtaining locations of trapped personnel quickly and accurately is the key to save more lives in mishaps and natural disasters such as earthquakes, fires, and straying in mountains. The industry is seeking to address this problem and improve the accuracy and efficiency of localization at scenes of disasters [1]. For example, UAVs were used in 2015 to search and rescue trapped people in the Yangtze River [2]. However, current UAV positioning approaches have not plan a flight path and they can only provide rough position estimation. This leads to time consumption and low accuracy significantly $[3,4]$. Rescues would benefit tremendously from timely and accurate localization $[5,6]$. For example, for an emergency rescue mission in the earthquake, many lives would be saved if the rescue is provided timely. Therefore, rescuers need to be aware of the positions of trapped persons quickly and accurately. Similarly, at the scene of fire, roughly knowing which floor trapped persons are located on is far from enough. It is better for rescuers to quickly and accurately know the exact locations of trapped persons, for example, whether or not the person is inside a specific room. In such case, we can rescue trapped persons timely and save many lives. In addition, fast and accurate positioning is also indispensable for some other emerging UAV applications. For instance, when someone is suddenly ill on a crowded street, if rescuers can find the exact position of the patient timely, the serious life threat of patient can be significantly reduced [7].

UAV positioning has recently been studied in the literature $[1,8-10]$. Using the signal of wireless devices, such as mobile phones [11], carried by targets to locate targets is the common approach in this domain. One method is to use the aerial sensor network [12, 13], SensorFly [1, 9], which locates targets through the multiple anchor nodes deployed on the ground communicating with the node on the UAV. 
The method needs to deploy anchor nodes in the field, but the deployment is difficult, expensive, and slow. Some other researchers propose to improve the localization accuracy by adopting multiple UAVs [10, 14-16]. However, this method requires the UAV to traverse the scanning area along a fixed flight path, which not only lacks flexibility, but also results in a longer searching path and a large amount of time and energy consumption. As another alternative, Warflying [1719] directs the UAV to fly to any point of the open space to detect and accurately locate wireless target devices $[8,20]$. Nevertheless, it needs to traverse the scanning target area according to the predetermined space filling curves no matter whether there exists a target or not [21], leading to low efficiency.

This paper introduces GuideLoc, a fast and accurate system for wireless localization of multiple targets based on the combination of applying UAVs and the method which uses received signal strength indicator (RSSI) and angle of arrival (AOA) to locate. Similar to current localization methods [22], GuideLoc uses RSSI [23] and AOA to estimate target positioning. Because the RSSIs sensed by the UAV from various directions are different and the direction in which the signal strength is the strongest corresponds to the direction of the target, the position of the target can be estimated. However, a key issue is that the localization is very rough and can generate serious positioning errors.

Different from these rough localization methods [24], GuideLoc controls the UAV to fly towards the target according to AOA information of the target. When GuideLoc arrives just over the target, it adopts its own GPS coordinates as the location data of the target, which can obtain the accurate location information. We propose a method which is mentioned in more detail subsequently to determine whether the UAV is over the target. At the same time, GuideLoc proposes the flight path planning algorithm for multitarget localization in an area. It needs to sequentially search each unit partition, calculate the flight path in each unit partition, and position each wireless target device in a unit partition along the flight path.

To illustrate GuideLoc [25], a simple example of aerial wireless localization is shown in Figure 1. Because the AOA and RSSI information of a target has certain deviation, it can cause inaccurate localization. Therefore, we need to adopt an advanced localization strategy to improve the accuracy. We allow the UAV to start from the starting point, fly along the planned path, and receive the wireless signal of each target device in real time during the flight process. When the UAV is over the target, the GPS coordinates of the UAV [26] are adopted as the location coordinates of the target device.

How can we determine whether the UAV is over the target device? The approach is to use the average of RSSIs. Specifically, if the average of RSSIs of the target is less than the threshold [24], the flight direction of the UAV needs to be adjusted for approaching to the target. Otherwise, it is deemed that the UAV has been over the target and the current GPS coordinates of the UAV are taken as the location coordinates of the target.

When the detection range of the UAV is smaller than the target area, how can the target detection $[4,27,28]$ be

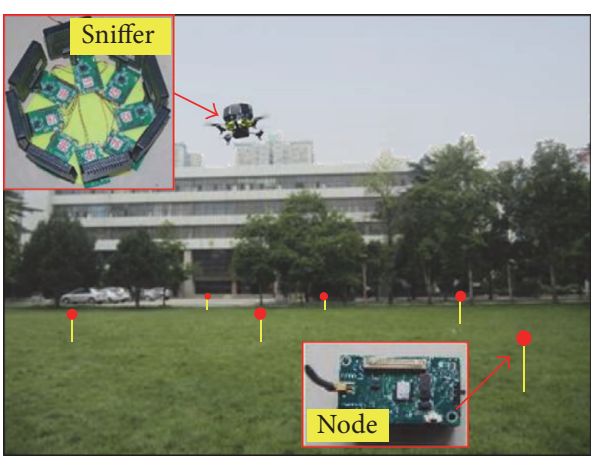

FIGURE 1: GuideLoc in localization.

accomplished in that area? To solve this problem, the whole area needs to be divided into a number of unit partitions [29]. Moreover, it should be noted that, in the real world, the targets are not evenly distributed in the target area. In some extreme cases, there is not any target device in some places.

In other cases, there could be multiple target devices in a unit partition. In order to complete the localization for all devices, the UAV will traverse the targets along a searching path. We present the design details in Section 5. Experiments verify the accuracy and rationality of the localization method in Section 7.

Summary of Experimental Results. We construct the GuideLoc system which consists of an ArduCopter with 8 wireless sniffers, a handheld controller, a software controller, and a target device locator. The performance of GuideLoc is evaluated using 12 wireless nodes deployed on our playground. In the experiments, we deploy 3, 6, 9, and 12 wireless nodes, respectively, as the targets and randomly distribute them on the football field. The transmission interval of the target is 1 second and the flight speed of the UAV is set to $5 \mathrm{~m} / \mathrm{s}$. In such condition, we compare GuideLoc with HAWK and obtain the following results:

(i) The positioning accuracy of GuideLoc can achieve 2.7 meters in average, which is slightly higher than that of HAWK;

(ii) The flight path length of HAWK is $2-5$ times as long as that of GuideLoc under the different densities of target nodes.

\section{Contribution}

(i) It puts forward the first positioning system which combines target guiding with regional division. The positioning accuracy of the system is 2.7 meters in average in the area which is a $1 \mathrm{~km} \times 1 \mathrm{~km}$ square, even in the case that targets are not evenly distributed in the area and wireless signal cannot cover the entire area.

(ii) The system can position targets in an area fast and accurately, which significantly reduces the power consumption of the UAV.

(iii) It proposes the averaging method for determining whether the UAV is over the target and verifies the 
feasibility of the method in practice through the experiments.

(iv) It also provides a regional division method constrained by the one-hop communication coverage of a wireless device and verifies the effectiveness of the method using NS2 to simulate the positioning process.

\section{Background}

UAVs are unmanned aircrafts manipulated by radio remote control apparatus and self-provided program-control devices. Instead of a cockpit, an autopilot and program-control devices and so forth are installed inside a UAV [30]. The staff on the ground carry out tasks such as tracking [31] and positioning, remote controlling, and data transmission [32] by radar or other equipment.

The wireless localization system based on UAV consists of five components, namely, a multirotor helicopter, wireless sniffers, a handled controller, a software controller, and a target device locator [33]. The localization process is such that we configure a UAV equipped with a searching and positioning system and control the UAV to fly along a certain path. Because a target device periodically transmits the radio at some particular frequency [34], the directional antennas installed on the UAV can be used to collect RSSIs, estimate the AOA of the signal according to the RSSIs, and subsequently get the direction information of the target [22]. In addition, in the case of multiple targets, different address codes in the packets can be used to distinguish the signals from different targets.

The method mentioned above for searching and rescuing based on UAVs exhibits several features which are particularly meaningful for the localization problem.

(i) The biggest advantage of using a UAV is that it can fly at low height and obtain image data under simple conditions, even in the harsh natural environment [35]. In this way, we can reduce the search scope and rescue scale without costing a great deal of time, manpower, and material resources, which improves the effectiveness of the rescue, lowers search cost, and enhances the safety of rescuers.

(ii) With regard to RSSI technology [36-38], because the detection equipment is not sophisticated and its hardware is of low cost, we can obtain a more accurate RSSI value through averaging RSSI values from multiple measurements and reduce the impact of multipath and shadowing effect.

(iii) AOA data can be obtained by an antenna array or combining several receivers [22], which is easy to be realized in technology and could also bring about high positioning accuracy due to little noises in angle estimation.

(iv) Different address codes in the packets sent by the different devices can be used to distinguish the signals from different targets.

\section{Overview}

GuideLoc is an aerial wireless localization system which uses target guiding based on region division and is also energyefficient. It can locate trapped targets quickly and accurately in the disaster rescue. Its flight distance is more than $50 \%$ shorter than that of other known wireless localization methods based on UAVs.

To realize the localization, GuideLoc collects wireless signals from the targets nearby. We have designed an experiment to simulate the disaster site in which 12 wireless nodes are randomly deployed as the targets to be searched on the football field. The transmission interval of the target signal is set to 1 second and the flight speed of the UAV is set to $5 \mathrm{~m} / \mathrm{s}$. GuideLoc presents the best searching path based on the genetic algorithm and uses wireless sniffers to collect RSSIs and AOA of a target. In addition, the GPS coordinates of the UAV are used to locate the target. As a result, GuideLoc achieves 2.7 meters of positioning accuracy on average. steps.

To locate a target, GuideLoc goes through the following

Step 1. If the target area is less than or equal to the one-top communication range of a wireless device, it will be regarded directly as a unit partition and the procedure goes to Step 2. Otherwise, the target area is divided into a number of unit partitions to ensure that each point in a unit partition can detect the signal from any other point and use the genetic algorithm to plan the optimal searching path for visiting each unit partition. (It will be explained in detail in Section 4.)

Step 2. Take the center of the nearest unit partition as the starting point of the UAV, fly to visit each unit partition, and detect the signals of wireless devices in real time to search targets in a unit partition.

Step 3. When the UAV arrives at a unit partition center, if GuideLoc can detect one or more new targets in its communication range, then go to Step 4; if not, it will determine whether it has finished the detection of all the unit partitions. If the detection has been finished, the localization process is over; if not, the UAV will fly to the center of the next unit partition along the searching path and repeat Step 3.

Step 4. GuideLoc obtains AOA and RSSI information of all targets, estimates the location of each target according to the information, uses the genetic algorithm to plan the optimal flight path for visiting each target with estimated locations, and then starts to traverse along the determined optimal path. (It will be illustrated in detail in Section 5.) When the UAV flies towards a target, the AOA and RSSIs of the target should be monitored in real time. The direction where signal strength is the strongest is considered as the direction of target. Then, the UAV is controlled to fly towards that direction. Afterwards, averaging method is used to judge whether the UAV is over the target; if yes, the GPS coordinates of the UAV are returned as position information of the target. (It will be explained in detail in Section 6.) 
Step 5. After the UAV determines locations of all the targets in the current unit partition, it will fly to the next unit partition according to the planed path in Step 1. Return to Step 3.

We will discuss steps above and related technology in more detail in the following sections. In Section 4, we mainly discuss the region division; in Section 5, we describe the planning of best path for target localization in the target area; and then in Section 6, we discuss how to use the averaging method to locate a target.

\section{Region Division}

When the target area is larger than the one-hop communication range of a wireless device, dividing the target area must be done, because GuideLoc first needs to estimate the locations of all targets according to the received RSSI and AOA information sent by targets in a one-hop communication range, then plans the flight path according to the estimated locations, and last traverses each target. When the UAV flies right over a target, GuideLoc will take the GPS coordinates of the UAV as the target location information.

The purpose of region division is to ensure that any unit partition can be covered by the one-hop communication range of a wireless device, which can make GuideLoc work efficiently in each unit partition. Obviously, the UAV traverses each unit partition and locates the targets within each partition, which can solve the positioning problem when the target area is larger than a one-hop communication range. Of course, in order to shorten the flight distance, GuideLoc needs to traverse each unit partition along a shortest path.

4.1. Region Division. The core task of region division is to determine the size of a unit partition. There are two main limitations on the size: (1) Each unit partition can be covered by the one-hop communication range of a wireless device; (2) When the UAV flies at the center of unit partition, it can have received at least one data packet sent by each target in the unit partition. As a consequence, the factors influencing the division include the size of the target area, the radius $r$ of a one-hop communication range, the UAV flight speed $v$, and the time interval $t$ of packet transmission of a target.

In order to explain the method of regional division easily and clearly, we assume that the target area is a square with $d$ on a side, and $N \times N$ unit partitions (the grid sections) are gotten after dividing the area, as shown in Figure 2. The essence of region division is to determine an optimal $N$. To ensure that GuideLoc can control the UAV to traverse all the targets in a unit partition on the basis of the real-time RSSI and AOA information, the UAV must receive a data packet sent by any other target anywhere in the unit partition, regardless of the position of the UAV in this partition. In other words, a unit partition is a subset of the intersection of all the one-hop communication ranges of targets in the partition. Because a unit partition is a square, the longest distance between any two points in the partition is the diagonal length. If the length is less than or equal to the radius $r$ of a one-hop communication range, the UAV can receive the data packets

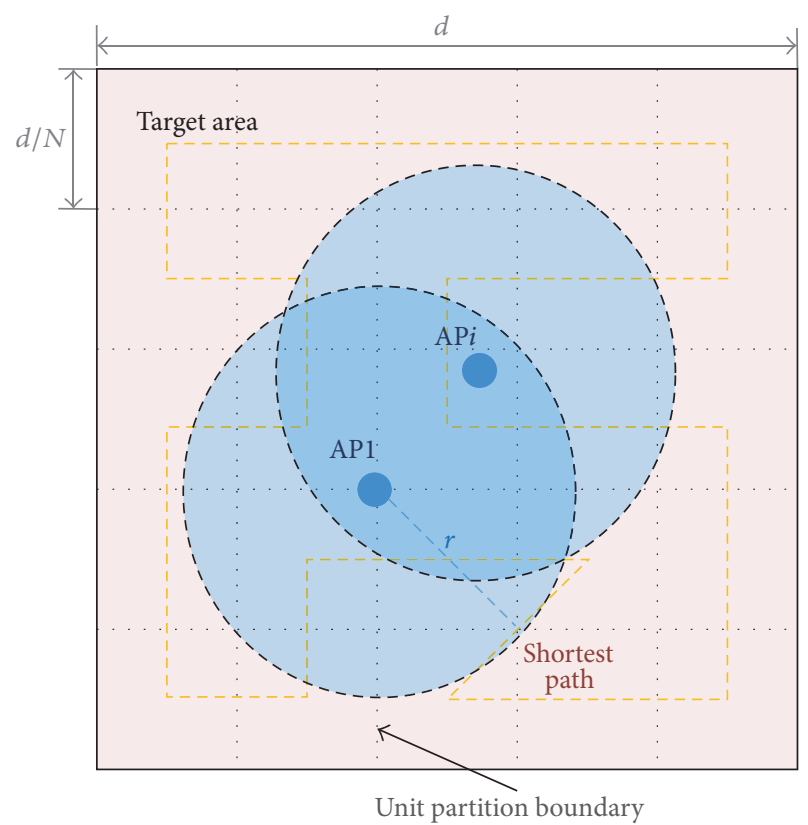

FIGURE 2: Illustration of regional division and shortest path for visiting every unit partition.

sent by any other targets in the unit partition, regardless of the UAV position in this partition. So the side length of a unit partition must satisfy

$$
\frac{d}{N} \leq \frac{\sqrt{2} r}{2}
$$

If $L$ represents the distance between the last traversed target in the previous unit partition and the center of the next partition, $L$ satisfies formula (2). And the minimum value of $L$ is obtained when the last traversed target in the previous partition is just at the center point of the adjacent edge of the previous and next partitions. It is $d / 2 N$. In order to ensure the UAV having traversed the last target in the previous partition can receive at least one packet from each target in the next unit partition before it reaches the center of the next partition, $L$ must fulfill formula (3)

$$
\begin{gathered}
L \geq \frac{d}{2 N}, \\
L>v \times t, \\
\frac{\sqrt{2} d}{r} \leq N<\frac{d}{v t} .
\end{gathered}
$$

Combining formulas (1), (2), and (3), formula (4) can be derived. In the actual division, the UAV is expected to fly at the fastest speed for search and rescue. Therefore, the optimum value of $N$ is $\sqrt{2} d / r$.

Discussion. A few points worth-noting are as follows:

(i) With regard to formula (3), since sending wireless signals is periodical [39], transmission interval $t$ of a 


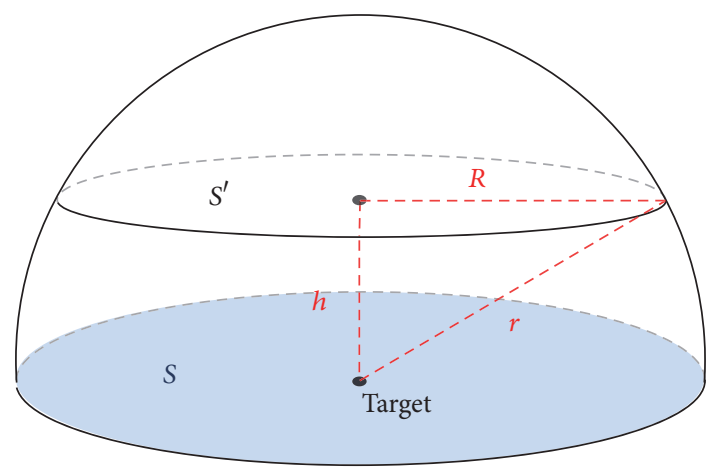

FIgURE 3: Effective range of UAV communicating with target.

target can be easily determined. The maximum flight speed of the UAV can also be set. Therefore, the minimum $L$ can be inferred and it is also ensured that the sniffer can detect all the mobile devices in the target region during the process of localization.

(ii) GuideLoc can search and locate targets in 2D space by a UAV. Because the UAV flies in the air and trapped targets with wireless devices are on the ground, when the UAV is flying at a certain height, the range where the wireless device on the ground can communicate with the UAV should be a circular cross section $S$, which is formed by the UAV flight plane cutting through the hemispheric communication range of the wireless device. The relationship between the radius $R$ of $S$, the UAV flight height $h$, and the radius $r$ of the wireless communication range is as shown in Figure 3 and the following equation:

$$
R=\sqrt{r^{2}-h^{2}} .
$$

4.2. Path Planning for Traversing All the Unit Partitions. After partitioning the area, GuideLoc needs to detect all the target devices in every unit partition so that all the targets in the whole area can be detected. Obviously, we should choose the shortest route for the UAV to traverse all the unit partitions and make sure that finally the UAV comes back to the start point after each unit partition is visited once and only once.

Planning the best searching path for all the unit partitions is the same as Traveling Salesman Problem (TSP). That is to say, the problem of flying path planning can be translated into TSP. In graph theory, this problem is to find out the Hamilton circle whose weight is the smallest in a given weighted connected undirected graph $G(V, E)$ and the vertexes of the graph $G$ are the center points of the unit partitions.

We make use of genetic algorithm (GA) commonly used to solve TSP as a solution to obtain the shortest flight path.

\section{Detection of Multitargets Inside a Unit Partition}

While the UAV traverses each unit partition, GuideLoc needs to detect all the target devices in each unit partition one by one so that all the targets in the whole area can be detected.
In order to improve the accuracy of localization, we require the UAV to fly right over the target to be detected and use the GPS coordinates of the UAV as the position information of the target. Because the UAV can detect all the targets in a unit partition, we can obtain AOA and RSSI values of these targets, estimate the location of every target in the unit partition according to the information, and utilize the location information to plan the optimal flight path for visiting every target in this unit partition. Thus, we need a proper method to plan this optimal path in a unit partition.

Intuitively, in a unit partition, the UAV should choose a route whose total distance is the shortest and traverse every target only once. Therefore, when we plan the shortest path in a unit partition, we need to guarantee that the UAV will not only go through all the targets, but also pass the each target only once in this unit partition and finally arrive at the center of next unit partition.

5.1. Determination of the Optimal Searching Path. The problem of planning the shortest flight path is similar to a TSP. However, a key challenge is how to translate the path planning into a TSP. The translating process is shown as follows.

The goal of TSP is to search for an optimal cycle for traversing $n$ points or to find a permutation $\pi(X)=$ $\left\{V_{1}, V_{2}, \ldots, V_{n}\right\}$ of the natural subset $X=1,2, \ldots, n$ (an element of the set $X$ represents a point number) to make formula (6) hold.

$$
\min T_{d}=\min \left\{\sum_{i=1}^{n-1} d\left(V_{i}, V_{i+1}\right)+d\left(V_{n}, V_{1}\right)\right\}
$$

In (6), $d\left(V_{i}, V_{i+1}\right)$ represents the distance between point $V_{i}$ and point $V_{i+1} . V_{1}$ is the starting point. But our problem is to find the shortest path to traverse all the points from the start to the end or search for a permutation $\psi(X)=$ $\left\{M_{1}, M_{2}, \ldots, M_{n}\right\}$ of the natural subset $X=1,2, \ldots, n$ (an element of the set $X$ represents a point number) and make formula (7) hold

$$
\min T_{d}=\min \sum_{i=1}^{n-1} d\left(M_{i}, M_{i+1}\right)
$$

In formula (7), $d\left(M_{i}, M_{i+1}\right)$ represents the distance between point $M_{i}$ and point $M_{i+1}, M_{1}$ is the starting point, and $M_{n}$ is the destination. Since this problem is similar to a TSP, we will use GA that is usually applied to solve a TSP to settle this problem.

Algorithm. $d\left(M_{n}, M_{1}\right)$ is added to formula (7); that is to say, a virtual edge from the starting point to the destination is added and $d\left(M_{n}, M_{1}\right)$ is set as a minimum negative number; thus

$$
\begin{aligned}
\min T_{d} & =\min \sum_{i=1}^{n-1} d\left(M_{i}, M_{i+1}\right)+d\left(M_{n}, M_{1}\right) \\
& =\min \left\{\sum_{i=1}^{n-1} d\left(M_{i}, M_{i+1}\right)+d\left(M_{n}, M_{1}\right)\right\} .
\end{aligned}
$$




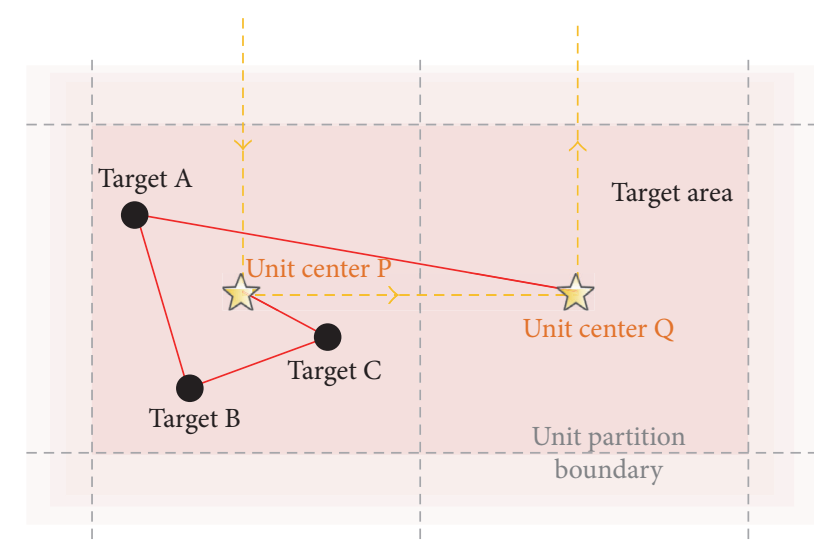

FIGURE 4: Shortest flight path within a unit partition.

Comparing formula (8) with formula (6), formula (8) is just the expression of TSP. Our problem can be converted into a TSP and it also belongs to a kind of NP complete problem.

In our algorithm, we first figure out the estimated positions of each wireless device using their RSSI and AOA values. Thereafter, we take the center of the current unit partition as the starting point of the searching path within this unit partition and a vertex of the graph $G^{\prime}$, take the center of the next unit partition in the region searching path as the destination of the searching path within this unit partition and a vertex of the graph $G^{\prime}$, take all the targets in the current unit partition as the other vertices of the graph $G^{\prime}$, and take the distance between any two vertices in $G^{\prime}$ as an edge weight in $G^{\prime}$. Finally we get a weighted connected undirected graph $G^{\prime}$. In order to guarantee that the smallest Hamilton circle worked out must include the edge from the starting point to the destination, this edge is assumed to exist in the graph $G^{\prime}$ and its weight is supposed to be a very small negative.

Finally, we make use of the genetic algorithm to find out a Hamilton circle whose total distance is the shortest in the weighted connected undirected graph $G^{\prime}$. Because the weight of the virtual edge between the starting point and the destination is assumed as a constant, after the virtual edge is deleted from the Hamilton circle having been found out, the rest of edges in the Hamilton circle just form the shortest path from the starting point to the destination through all the points.

That is to say, the path is the shortest flight path within a unit partition, for example, the path $\mathrm{P}-\mathrm{C}-\mathrm{B}-\mathrm{A}-\mathrm{Q}$ expressed using a solid red line as shown in Figure 4.

\subsection{Determination of the Unit Partition Corresponding to the} Target. When we apply the genetic algorithm to complete the localization of all the targets, a practical challenge arises: which unit partition does a target device belong to on earth?

After a region has been divided into some partitions, it is apparent that the positions of all the target devices located in this region are also determined. The distance between a target device and the center of the current unit partition is calculated using the RSSI values of the target device based on Log-Normal Shadowing Model (LNSM) [40]. If the distance

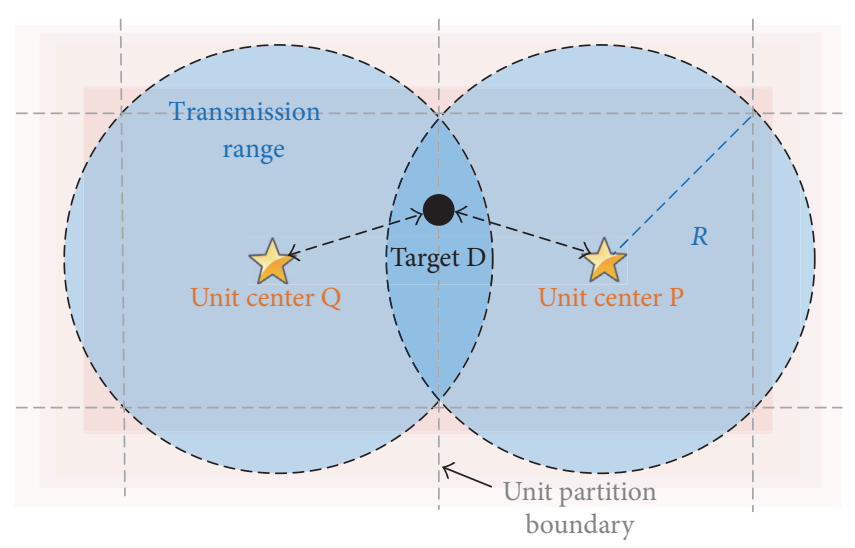

Figure 5: Illustration of target on the boundary between two adjacent unit partitions.

is less than or equal to the threshold, the target device will belong to the current unit partition. Otherwise, it will not belong to this unit partition. However, since there exists a large error in the estimated distance according to the RSSI, a target device may be missed to be located due to the possible mistake made in determining which unit partition the target device belongs to.

The point $\mathrm{D}$, for example, is on the boundary between two adjacent unit partitions in Figure 5. When the UAV arrives at the center of the right unit partition, if the estimated distance is longer than the actual distance, the UAV will judge that D does not belong to the unit partition.

Similarly, when the UAV arrives at the center of the left unit partition, if the estimated distance is slightly longer than the actual distance, the UAV also decides that it does not belong to the unit partition, which results in missing the detection of target device $\mathrm{D}$.

In order to avoid missing any target, a threshold $\lambda$ is set as the half of the diagonal length of a unit partition as formula (9) shown below:

$$
\lambda=\frac{\sqrt{2} d}{2 N}
$$

Then we can get a circular decision region. Its center is the center of a unit partition and its radius is the threshold. Apparently, missing a target device can be effectively avoided because the boundaries of any unit partition are all included inside a decision region.

Specifically, when the UAV approaches to the center of the right unit partition and makes use of RSSI values of the target device $\mathrm{D}$ to estimate the distance, even though the estimated distance is longer than the real distance, it would not give an erroneous judgment and can avoid missing the target D because $\mathrm{D}$ is still in the circular decision region.

Since the GuideLoc can mark the targets having been localized with their identification (i.e., their IP addresses), a target in an overlapping area between the two adjacent decision regions would not be localized more than once. 


\section{Single Target Localization}

After determining the path of visiting each target in a unit partition, the GuideLoc will realize the fast and accurate positioning for a single target and then complete the accurate positioning for all the targets in the unit partition.

In many applications, reducing the complexity of data collection is required so as to position the target quickly and accurately. In this case, we need to estimate a target location by using a number of directional antennas to receive RSSI and AOA data of the target and utilizing a RSSI-based ranging method. However, the ranging method based on RSSI is a very rough positioning technology which could give rise to serious positioning errors.

For instance, when the geographical factors of the detection area remain constant, the main factors affecting RSSI values are the nongeographical factors, such as temperature, humidity, and air pressure. The effect of changes of such factors on the wireless signal transmission is ruleless. The fluctuation caused by them for ranging based on RSSI is not very significant. But they can cause greater impact on the distance estimation for a target whose propagation model has been fixed, result in RSSI-based ranging error becoming larger, and thus affect the positioning accuracy. Instead, GuideLoc uses the following method in order to finish the positioning accurately.

Method. GuideLoc controls the UAV to fly towards and gradually approach a target according to the angle information of the target. When the UAV reaches the point just right over the target, the GPS coordinates of the UAV are used as the position coordinates of the target.

Nevertheless, this method brings about a new challenge: how to determine whether the UAV has been just right over the target or not.

We designed two methods for this challenge. One is range method. The basic idea is as follows: when the UAV is exactly right over a target, ideally, the RSSIs from different directions almost all are equal, which means the range of the RSSI values closes to 0 . But the experiment shows that it is not better than the averaging method. The idea of averaging method is as follows: when the UAV flies into the communication range of a target, it will receive wireless signals sent by the target. The RSSIs in different directions vary. In theory, the direction in which signal strength is the strongest is the direction of the corresponding target. Thus the UAV can arrive at the point which is just right over the target by flying towards the target direction. When the UAV flies towards a target and approaches it, the RSSI will gradually increase. The RSSI is the strongest when it is closest to the target. But it will gradually decrease when the UAV flies away from the target. Therefore, we can use the average of RSSIs to determine whether the $\mathrm{UAV}$ is just right over the target. The formula is

$$
\overline{\mathrm{RSSI}}=\frac{1}{n} \sum_{i=1}^{n} \mathrm{RSSI}_{i},
$$

where $n$ is the number of antennas installed on the UAV ( $n$ is 8 in GuideLoc), $i$ is an antenna label, and RSSI ${ }_{i}$ is the RSSI sensed by the antenna in $i$ direction.

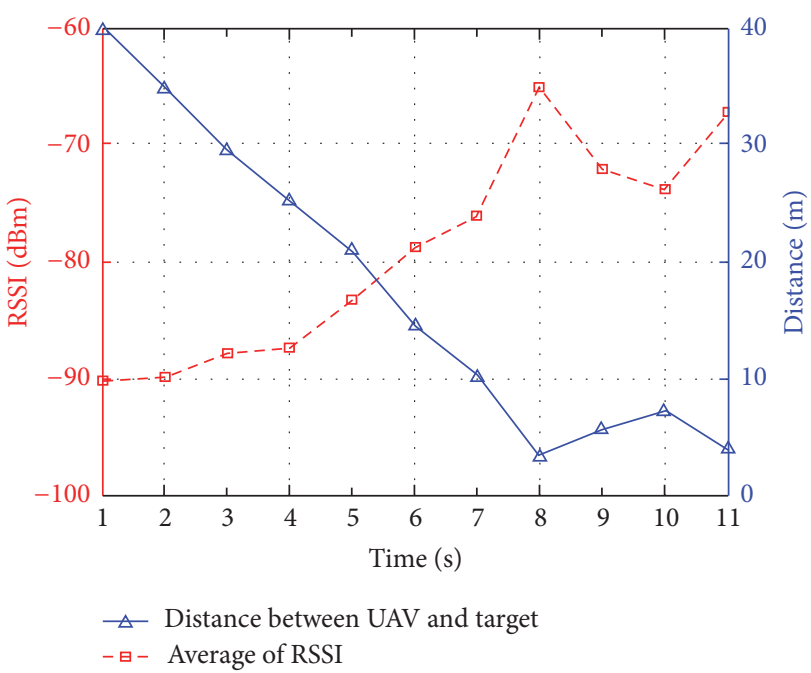

FIGURE 6: The result of the averaging method experiment.

In order to validate the feasibility of the averaging method in practice, an experiment is conducted with the related data used in the above experiments. The result is shown in Figure 6 where the blue line represents the change of the distance between the UAV and the target and the red line represents the change of the average of RSSIs. The figure shows that the positioning process can be divided into three stages. In the first stage ( $1 \mathrm{~s}-8 \mathrm{~s}$ ), when the UAV flies towards and gradually approaches the target, the average of RSSIs gradually increases. In particular, the average of RSSIs reaches the maximum when the UAV is the closest to the target $(8 \mathrm{~s})$; in the second stage ( $8 \mathrm{~s}-10 \mathrm{~s}$ ), the average of RSSIs gradually decreases as the UAV flies away from the target. In the last stage (10 s-11s), as a result of only one target node deployed in the experiment, the UAV hovers around the target, which brings about a gentle fluctuation of the average of RSSIs. Therefore, the averaging method is feasible in practice to determine whether the UAV is just right over a target or not.

To reduce the energy consumption of the UAV, a threshold needs to be used as a key factor in the aerial wireless location algorithm based on target guiding. Specifically, GuideLoc takes a target as the next destination and controls the UAV to fly towards it. During the flight, the UAV constantly captures the wireless signals transmitted from the target and gets the new data. The average of RSSIs is subsequently calculated. If the average is less than the threshold, GuideLoc will update the direction information, adjust its flight direction, and continue to fly close to the target. Otherwise, the UAV is regarded as having been just right over the target, and the current position of the UAV is served as the position of the target. Then the current GPS coordinates of the UAV are transmitted to a Lenovo laptop X230 on the ground by an XBee wireless transceiver and the target positioning is completed.

The location algorithm based on target guiding is as shown in Algorithm 1. 
(1) Get measurement samples of the target;

(2) Estimate AOA according to RSSI of different directions;

(3) GuideLoc flies towards the target;

(4) Capture new measurement samples of the target;

(5) Use Formula (10) to calculate the average of RSSIs;

(6) if (The average of RSSIs $<-70$ )

(7) Update AOA, adjust the direction of flight and

(8) continues flying close to the target;

(9) else

(10) Arrive just right over the target, Locinfo = GPS;

(11) endif

(12) return Locinfo.

Algorithm 1: Algorithm of GuideLoc.

\section{Discussion}

(i) A threshold is used to determine whether the UAV is just over the target or not in the averaging method. The threshold setting is associated with the devices used. Different devices have their respective thresholds. The threshold is set to $-70 \mathrm{dBm}$ in our experiment.

\section{Experiment and Analysis}

After obtaining the division algorithm for determining the unit partition size and the target positioning algorithm, we need to verify their validity and feasibility and evaluate their performance. Firstly, we verify whether the unit partition size meets formula (4) through simulation and, thereafter, compare the performance of GuideLoc with that of HAWK using different densities of target nodes. Through the experiments, we obtain the positioning performance of the target wireless devices in the playground of our campus. However, since disaster rescue is often expected as fast as possible in real life, the effects of flight speed on the performance of the system also need to be studied.

7.1. Simulation Analysis. To verify whether the unit partition size is consistent with formula (4), we use NS2 to simulate the positioning process. Firstly, we configure the transmission range of a target wireless device as $300 \mathrm{~m}$, the broadcast interval of a packet as $1 \mathrm{~s}$, the flight speed of the UAV as $10 \mathrm{~m} / \mathrm{s}$, and the target area as a square area of $1000 \mathrm{~m} \times 1000 \mathrm{~m}$ in which 30 wireless nodes are randomly deployed.

In the simulation, the UAV flies and searches along the unit partitions initially according to the optimal Hamilton circle. When a packet from a wireless node is received by the UAV, the distance between the node and the UAV is recorded. If the range of the RSSI values in different directions is not greater than $11 \mathrm{dBm}$, the current position of the UAV is taken as the position of the node and the distance between the node and the UAV is regarded as the positioning error. Subsequently, we use $0,10,20,30, \ldots, 190,200$ wireless nodes which are randomly and evenly distributed in the square area and run the simulation 30 times under each node density.

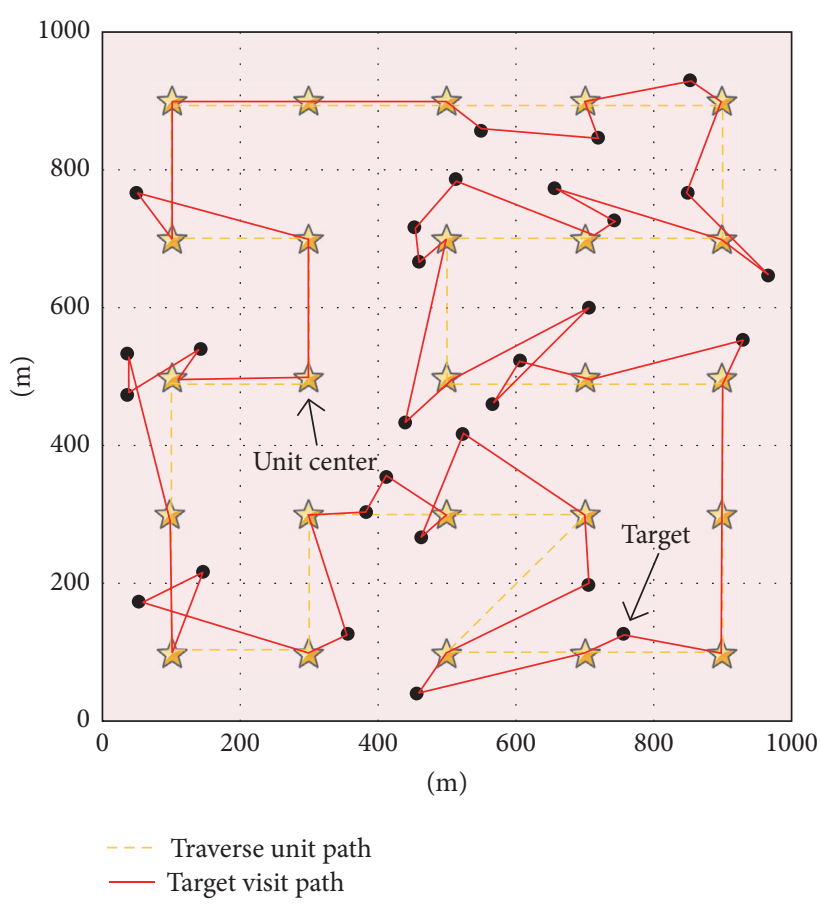

FIGURE 7: Flight route of GuideLoc: unit traversal path shows the traversal order of unit partitions and target visit path shows the path of visiting targets in each unit partition.

We apply the above simulation configuration to formula (4) to acquire the size of square unit partition. The target area is thus divided into $5 \times 5$ unit partitions as shown in Figure 7 . The red solid line in the figure illustrates the flight path of GuideLoc in the positioning process. The UAV searches 25 unit partitions in order, so that it can locate the 30 mobile devices in the target area. At macrolevel, the UAV searches all the unit partitions along the smallest Hamilton circle (the yellow dashed line). At macrolevel, after the UAV flies through the center of the unit partition, it will fly towards the first target to be visited.

The simulation results are presented in Figure 8 where there are two CDF curves, respectively, representing the positioning errors of GuideLoc and HAWK under the 21 groups 


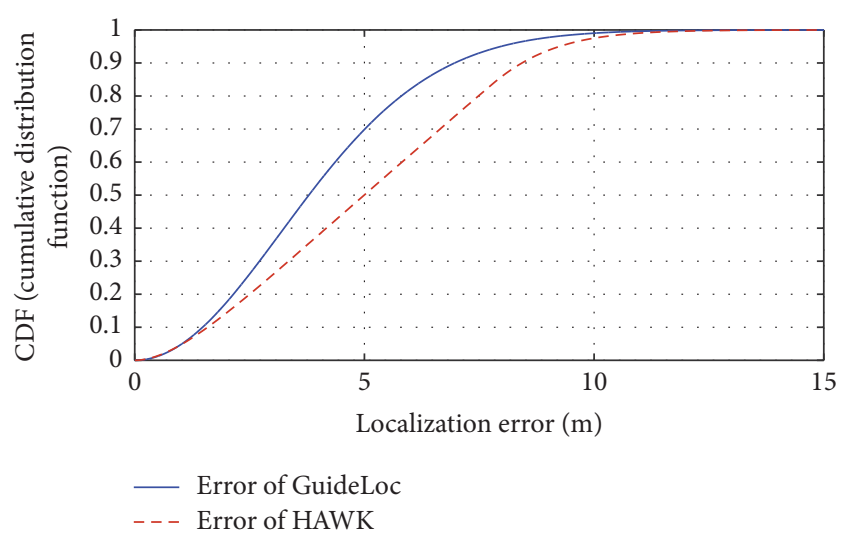

FIGURE 8: Localization errors of GuideLoc and HAWK.

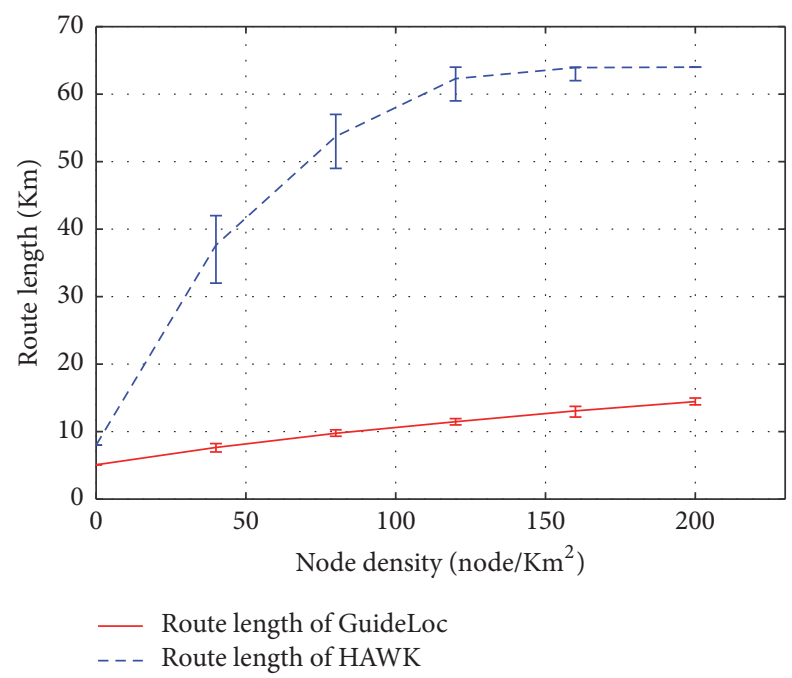

FIGURE 9: Flight path length of GuideLoc and HAWK under different node densities.

of node densities. The figure shows that the positioning accuracy of GuideLoc is slightly higher than that of HAWK. The median of the positioning error of GuideLoc is about $4 \mathrm{~m}$, while that of HAWK is about $5 \mathrm{~m}$. Moreover, the maximum error of GuideLoc is less than $12 \mathrm{~m}$, while that of HAWK is less than $15 \mathrm{~m}$.

In Figure 9, the length of the flight paths of GuideLoc and HAWK under the different node densities is shown. The length of the flight path of GuideLoc is obviously much shorter than that of HAWK. Under each node density, the length of the flight path of HAWK is 2-5 times as long as that of GuideLoc. When the node density is 0 (that is to say, there is not any target device in the target area), the flight path of HAWK is a level 3 Moore curve, the length of which is $8000 \mathrm{~m}$, while the flight path of GuideLoc is the smallest Hamilton circle, the length of which is only $5082 \mathrm{~m}$. The length of the flight paths in two methods is increasing with the increment of node deployment density, but the path length of HAWK increases quickly and the path length of GuideLoc increases slow.

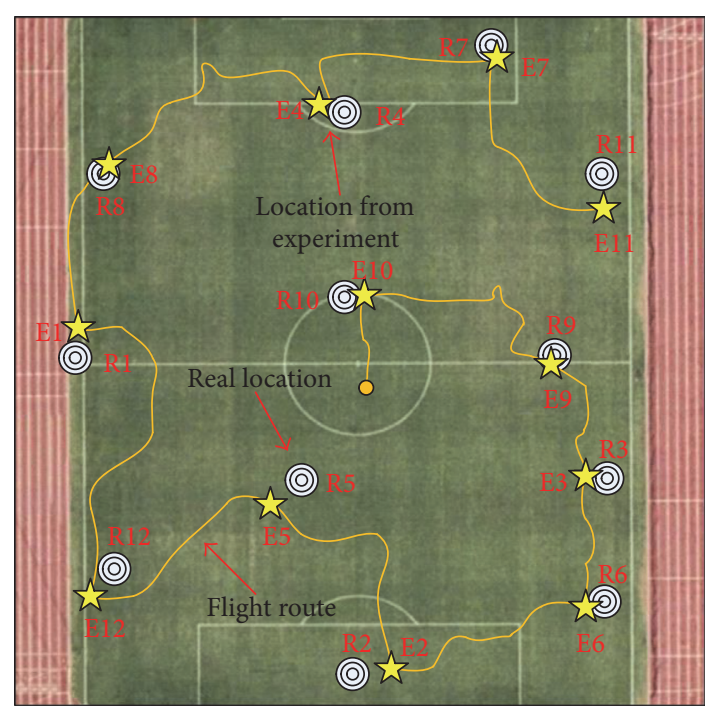

Figure 10: APs identified by GuideLoc.

7.2. Experiment Analysis. In this section, we obtain the results of wireless devices localization in the campus playground through real experiments and use them to evaluate the performance of GuideLoc. In the experiment, 3, 6, 9, and 12 wireless nodes are randomly distributed on the football field, respectively. We denote the beacon transmission interval of the target by $t$, which is 1 second. The flight speed of the UAV is set as $5 \mathrm{~m} / \mathrm{s}$. In order to ensure the positioning accuracy of HAWK and GuideLoc is on the same order of magnitude, a level 3 Moore curve is used as the flight path of HAWK.

However, because the UAV battery can only power its flight for about 15 minutes, a large scale experiment is not able to be conducted.

The experiment results are shown in Figure 10 which not only indicates the flight path of GuideLoc (the yellow solid line) in the localization process in the case of 12 targets deployed, but also marks the real and estimated locations of those targets (the white dot represents the real location; the yellow star represents the estimated location). Figure 11 shows the CDF curves of localization errors of GuideLoc and HAWK, respectively, in the case of different number of nodes. GuideLoc can achieve the accuracy of 2.7 meters in average. Figure 12, respectively, shows the total length of the flight paths of GuideLoc and HAWK in the case of different number of nodes. The proposed method makes use of the direction information of a target device so that the UAV can fly directly towards the target along the shortest flight path. As expected, when compared with HAWK, GuideLoc can significantly reduce the total flight distance and save the power consumption of the UAV.

We also need to evaluate the impacts of various flight speeds on the localization accuracy and time cost. For this purpose, in the experiment, only one wireless target node is deployed on the football field. The packet transmission interval of the node is set to 1 second. GuideLoc takes off from the edge of the football field to locate the target node and its 


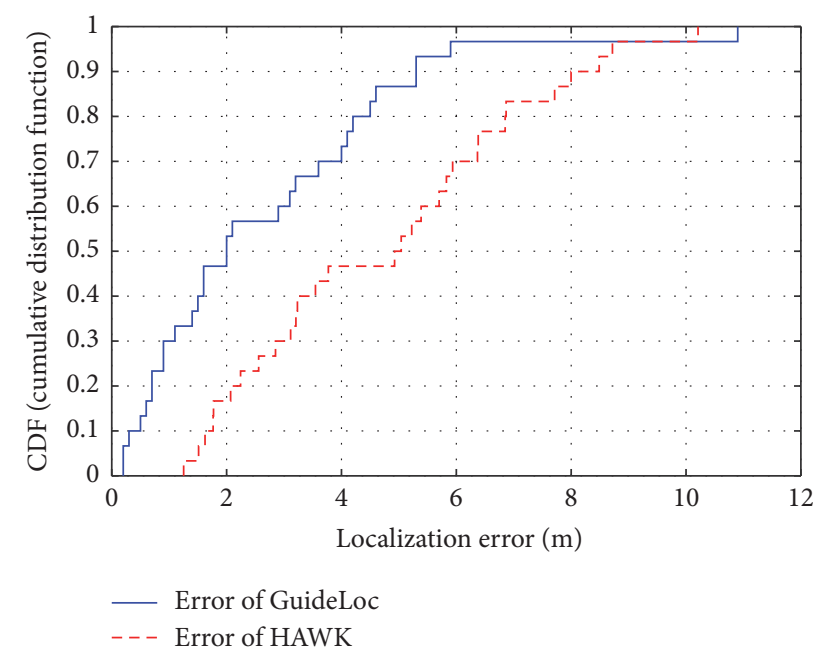

FIGURE 11: Localization errors of GuideLoc and HAWK.

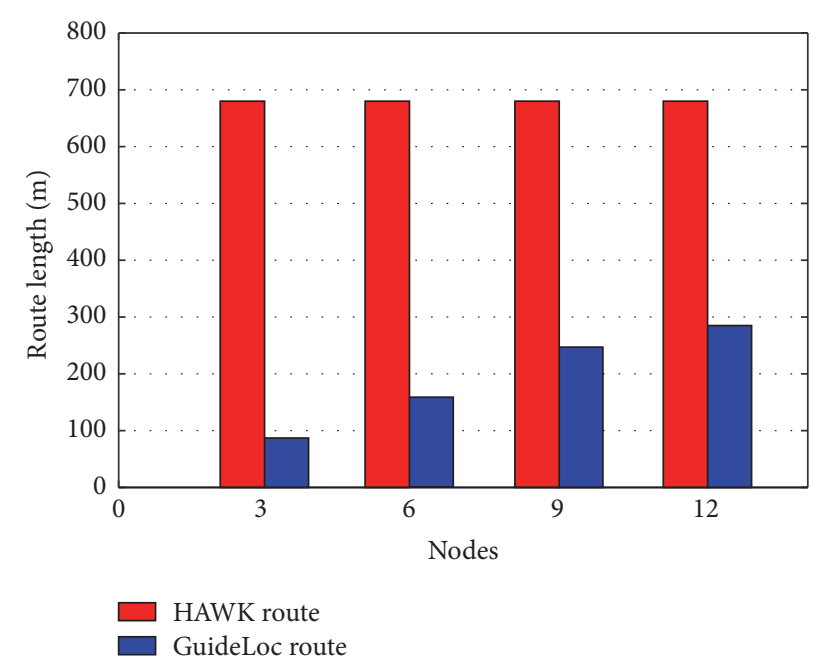

FIGURE 12: Route length of GuideLoc and HAWK.

flight speed is, respectively, set to $1,2,310$, and $11 \mathrm{~m} / \mathrm{s}$. The experiment is repeated ten times under each flight speed.

Figure 13 shows the localization error and time consumption of GuideLoc under the 11 different flight speeds. As expected, the faster the flight speed, the less the number of measured samples and thus the greater the localization error. However, the faster the flight speed, the less the consumed the localization time and thus the higher the efficiency of search and rescue.

In short, the flight speed has an obvious impact on the performance of GuideLoc, and the localization error and time consumption are two contradictory parameters, which need to be carefully balanced.

Therefore, it is crucial to analyze and quantify the application requirements of users, so as to set the matching flight speed and related performance parameters.

7.3. Experiment Summary. We firstly verify the formula which restrains the unit partition size by the simulation and

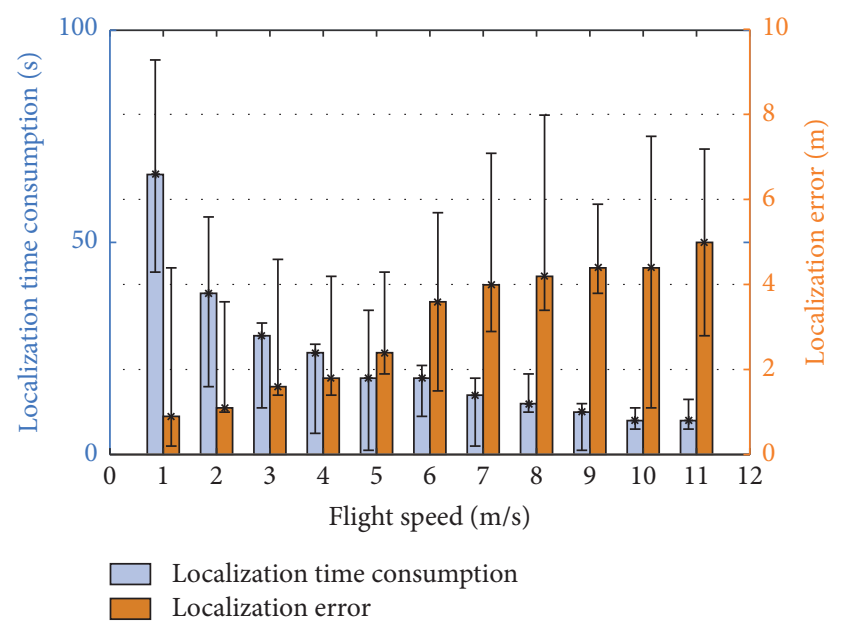

FIGURE 13: Localization error and time consumption of GuideLoc under the 11 different flight speeds.

then compare the performance of GuideLoc and HAWK under the different densities of target nodes. Finally, we conduct the real-world experiments and acquire the corresponding results of wireless devices localization on the football field. The simulation results indicate that the localization accuracy of GuideLoc is slightly higher than that of HAWK and the length of the flight path of HAWK is $2-5$ times as long as that of GuideLoc under the different densities of target nodes. The experiment results demonstrate that the localization accuracy of GuideLoc is 2.7 meters in average.

In summary, GuideLoc can directly fly towards a target device using the direction information of the target. Compared with HAWK utilizing space filling curves to traverse and scan, GuideLoc greatly reduces the total flight distance and saves the power consumption of the UAV.

\section{Related Work}

The early localization technologies for trapped targets in disaster rescue rely on Wardriving $[8,41]$ to collect RSSIs including direction information. Usually, the gradient information inferred from RSSIs is used to determine the location of an access point (AP) [20]. However, the technologies based on Wardriving are all limited by roads since it is difficult to reach some places, such as the top of high-rise buildings [29]. Thus the portability and flexibility of the system using this kind of technology are very limited.

Some researchers proposed heuristic rules (cluster [10]) based on the behavior of biological colony to establish an indoor cluster system and make a group of UAV s control their own flight. Each cluster member in the system is equipped with an airborne computer and a mini wireless camera [42], so multiple views can be collected to analyze through only one flight. Nevertheless, a common challenge for these technologies is that the localization effect is influenced by the cooperation effectiveness of these cluster members, that is, UAVs. Besides, it is very difficult to find out a hidden target in a practical application. 
HAWK [19] uses a space filling curve as a searching path and determines the level of searching path according to the UAV flight speed, the packet transmission interval of target, and the wireless transmission range in order to ensure that all the wireless devices in a search area can be detected [43]. However, it lacks flexibility, since it must traverse and scan a target area along a fixed space filling curve, regardless of whether there are targets in the area, which results in a longer searching path and a significant amount of time and energy consumption.

The technology of searching and locating targets by using UAVs has some limitations. Firstly, one or more helicopters are used to form a fixed space filling curve instead of planning an optimal searching path in advance. Secondly, the location information of targets is obtained by cameras or RSSIs of sensors. Using cameras to get location information usually needs human involvement. In addition, it is of slow speed and easy to miss some targets. Furthermore, the localization accuracy cannot be guaranteed and targets that are only in sight can be monitored. RSSIs can only help us roughly estimate the target location; thus the error is significant.

In contrast, GuideLoc can divide a target area into a number of unit partitions in advance and use the genetic algorithm to plan an optimal searching path for traversing each unit partition. Then it uses the UAV to locate a target and takes the GPS coordinates of the UAV as the target location information. This method not only improves the localization accuracy, but also uses only one UAV to search and locate all the targets along an optimized searching path.

GuideLoc is related to a lot of technologies in locating wireless devices.

Firstly, the technology of collecting wireless signals is adopted for RSSI and AOA measurement. In some conditions, only one rotating directional antenna is used to measure AOA. GuideLoc also uses directional antennas, but it employs a number of fixed directional antennas. Each antenna is connected to a radio receiver, which enables the UAV to receive the wireless frames from all directions at the same time.

In addition, the common method for getting the location of wireless devices is to install a camera or a mini DVR on a searching device or use RSSIs to estimate [44]. Different from these methods, GuideLoc controls the UAV to fly just right over a target by estimating the target location based on RSSI and AOA and takes the current GPS coordinates of the $\mathrm{UAV}$ as the target location information, which can improve the localization accuracy.

Furthermore, in order to further improve the localization accuracy, the data in the digital compass on the UAV is used to enhance the calculation. Then the collected information composes a measurement sample 〈target identification, GPS coordinates, UAV direction, antenna label, signal strength $\rangle$, which is stored into flash memory for future calculation.

Finally, the current methods for searching and locating targets using UAVs are always dependent on space filling curves to determine the flight path of the UAV [45]. However, GuideLoc divides a target area into a number of unit partitions in advance and uses the genetic algorithm to plan an optimal searching path to traverse each unit partition, which shortens the flight distance and lowers the energy consumption.

\section{Concluding Remarks}

Nowadays, rescue in disaster such as earthquake rescue, expedition trapped, travelers lost, fire rescue all makes use of wireless devices to locate the trapped victims. However, most of the current search and rescue techniques are based on the auxiliary functioning of facilities or training, which causes low localization accuracy and large energy consumption. With regard to Wardriving, such kind of technology only applies to empty areas such as grasslands in which there are fewer and shorter obstructions. It does not deal with areas with more and higher coverings such as mountains and buildings. As for Warflying, it requests a UAV to traverse and scan a target area along a fixed flight path, even if there is no target in the area. Apparently, it lacks flexibility and leads to a longer flight path which results in large time and energy consumption, which could further lead to some bad consequences. For example, if the searching time is too long, the best rescue time may be missed and the lives of persons in distress will be endangered. Furthermore, because of the complex environment of disaster area and adverse communication, medical treatment, climate and topography, the high energy consumption and long searching time will affect the search and rescue greatly. Facing with the conditions of trapped persons' lives and the complex environment, if we rely on UAVs to search and locate them, the localization system that caters our needs must satisfy shorter time, lower energy consumption, and higher accuracy. However, the current localization systems cannot accomplish that task.

GuideLoc presents a localization method for search and rescue, which is not only fast, but also energy-saving and accurate. It can improve the efficiency of search and rescue tasks. The basic idea of our proposed method is to plan a traversing path after the target region division estimates the targets location based on RSSI and AOA and use the GPS coordinates of the UAV as that of the target when the UAV flies just right over the target.

We believe that GuideLoc is able to advance the search and rescue technology in natural disasters, field investigation, geological exploration, and other special field, provide technical support for saving lives timely and accurately, and guarantee the rescuers' safety to some extent. At the same time, it provides a new paradigm for searching and locating persons in distress by using UAVs.

\section{Disclosure}

Anwen Wang and Xiang Ji should be considered co-first authors.

\section{Competing Interests}

The authors declare that there is no conflict of interests regarding the publication of this paper. 


\section{Authors' Contributions}

Anwen Wang and Xiang Ji contributed equally to this work.

\section{Acknowledgments}

This work was supported in part by the National Natural Science Foundation of China (no. 61572402, no. 61272461, no. 61602382, no. 61272120, and no. 61602381) and the Science Foundation of Northwest University (no. 15NW32 and no. 15NW31).

\section{References}

[1] A. Purohit and P. Zhang, "Sensorfly: a controlled-mobile aerial sensor network," in Proceedings of the 7th ACM Conference on Embedded Networked Sensor Systems (SenSys '09), pp. 327-328, Berkeley, Calif, USA, November 2009.

[2] H. Zhou, "Research on visible light shooting method for transmission line inspected by unmanned aerial vehicle," New Technology \& New Products of China, vol. 18, pp. 9-10, 2015.

[3] A. A. Heidari and R. A. Abbaspour, "Autonomous UAV path planning for search and rescue missions in post natural disaster assessment based on novel G-BFOA algorithm," Journal of Geomatics Science and Technology, vol. 3, no. 4, pp. 41-52, 2014.

[4] Z. Wang, H. Chen, Q. Cao, H. Qi, Z. Wang, and Q. Wang, "Achieving location error tolerant barrier coverage for wireless sensor networks," Computer Networks, vol. 112, pp. 314-328, 2017.

[5] J. Wang, D. Fang, Z. Yang et al., "E-HIPA: an energy-efficient framework for high-precision multi-target-adaptive device-free localization," IEEE Transactions on Mobile Computing, vol. 16, no. 3, pp. 716-729, 2016.

[6] J. Wang, X. Chen, D. Fang, C. Q. Wu, Z. Yang, and T. Xing, "Transferring compressive-sensing-based device-free localization across target diversity," IEEE Transactions on Industrial Electronics, vol. 62, no. 4, pp. 2397-2409, 2015.

[7] N. C. Sina, "Shanghai global carnival," 2003 http://news.sina .com.cn/c/2003-07-02/03581259997.shtml.

[8] A. P. Subramanian, P. Deshpande, J. Gao, and S. R. Das, "Driveby localization of roadside WiFi networks," in Proceedings of the 27th IEEE Communications Society Conference on Computer Communications (INFOCOM '08), pp. 718-725, Phoenix, Ariz, USA, April 2008.

[9] A. Purohit, Z. Sun, F. Mokaya, and P. Zhang, "SensorFly: controlled-mobile sensing platform for indoor emergency response applications," in Proceedings of the 10th ACM/IEEE International Conference on Information Processing in Sensor Network (IPSN '11), pp. 223-234, ACM/IEEE, Chicago, Ill, USA, April 2011.

[10] O. Holland, J. Woods, R. De Nardi, and A. Clark, "Beyond swarm intelligence: the ultraswarm," in Proceedings of the 2005 IEEE Swarm Intelligence Symposium, SIS 2005, pp. 225-232, Pasadena, Calif, USA, June 2005.

[11] Y. Zou, G. Wang, K. Wu, and L. M. Ni, "SmartScanner: know more in walls with your smartphone!", IEEE Transactions on Mobile Computing, vol. 15, no. 11, pp. 2865-2877, 2016.

[12] G. Wang, S. Zhang, K. Wu, Q. Zhang, and L. M. Ni, “TiM: finegrained rate adaptation in WLANs," IEEE Transactions on Mobile Computing, vol. 15, no. 3, pp. 748-761, 2016.
[13] H. Luo, K. Wu, Y. Gong, and L. M. Ni, "Localization for drifting restricted floating ocean sensor networks," IEEE Transactions on Vehicular Technology, vol. 65, no. 12, pp. 9968-9981, 2016.

[14] P. B. Sujit and D. Ghose, "Search using multiple UAVS with flight time constraints," IEEE Transactions on Aerospace and Electronic Systems, vol. 40, no. 2, pp. 491-509, 2004.

[15] S. V. Spires and S. Y. Goldsmith, Exhaustive Geographic Search with Mobile Robots Along Space-Filling Curves, Springer, Berlin, Germany, 1998.

[16] J. Scherer, S. Yahyanejad, S. Hayat et al., "An autonomous multiUAV system for search and rescue," in Proceedings of the 1st ACM Workshop on Micro Aerial Vehicle Networks, Systems, and Applications for Civilian Use (DroNet '15), pp. 33-38, ACM, Florence, Italy, May 2015.

[17] Wikipedia, "Warflying[eb/ol]," 2013 https://en.wikipedia.org/ wiki/Warflying.

[18] R. Queen, "Wireless aerial surveillance platform [eb/ol]," 2014 https://rabbit-hole.org/.

[19] Z. Liu, Y. Chen, B. Liu, C. Cao, and X. Fu, "HAWK: an unmanned mini-helicopter-based aerial wireless kit for localization," IEEE Transactions on Mobile Computing, vol. 13, no. 2, pp. 287-298, 2014.

[20] F. Comtech, "802.11 phocus array antenna system by fidelity comtech," 2014 http://www.fidelity-comtech.com/.

[21] V. De Araujo, A. P. G. S. Almeida, C. T. Miranda, and F. De Barros Vidal, "A parallel hierarchical finite state machine approach to UAV control for search and rescue tasks," in Proceedings of the 11 th International Conference on Informatics in Control, Automation and Robotics (ICINCO '14), pp. 410-415, SCITEPRESS, Vienna, Austria, September 2014.

[22] Y. S. Lee, J. W. Park, and L. Barolli, "A localization algorithm based on AOA for ad-hoc sensor networks," Mobile Information Systems, vol. 8, no. 1, pp. 61-72, 2012.

[23] G. Wang, Y. Zou, Z. Zhou, K. Wu, and L. Ni, "We can hear you with wi-fi!," IEEE Transactions on Mobile Computing, vol. 15, no. 11, pp. 2907-2920, 2016.

[24] H.-H. Hsu and C.-C. Chen, "RFID-based human behavior modeling and anomaly detection for elderly care," Mobile Information Systems, vol. 6, no. 4, pp. 341-354, 2010.

[25] S. Chen, D. Fang, X. Chen, T. Xia, and M. Jin, "Aerial wireless localization using target-guided flight route," in Proceedings of the ACM Conference on Special Interest Group on Data Communication (SIGCOMM '14), pp. 587-588, Chicago, Ill, USA, August 2014.

[26] Y. Kim, S. Lee, S. Lee, and H. Cha, "A GPS sensing strategy for accurate and energy-efficient outdoor-to-indoor handover in seamless localization systems," Mobile Information Systems, vol. 8, no. 4, pp. 315-332, 2012.

[27] Z. Wang, J. Liao, Q. Cao, and H. Qi, "Barrier coverage in hybrid directional sensor networks," IEEE Transactions on Mobile Computing, vol. 13, no. 7, pp. 222-230, 2013.

[28] Z. Wang, J. Liao, Q. Cao, H. Qi, and Z. Wang, "Friendbook: a semantic-based friend recommendation system for social networks," IEEE Transactions on Mobile Computing, vol. 14, no. 3, pp. 538-551, 2015.

[29] P. Egglestone, D. Ansell, and C. Cook, "DEMO: uavs in crowd tagged mountain rescue," in MindTrek, p. 286, ACM, New York, NY, USA, 2013.

[30] T. Tomic, K. Schmid, P. Lutz et al., "Toward a fully autonomous UAV: research platform for indoor and outdoor urban search and rescue," IEEE Robotics and Automation Magazine, vol. 19, no. 3, pp. 46-56, 2012. 
[31] X. Liu, M. Dong, K. Ota, L. T. Yang, and A. Liu, “Trace malicious source to guarantee cyber security for mass monitor critical infrastructure," Journal of Computer \& System Sciences, 2016.

[32] A. Liu, Q. Zhang, Z. Li, Y. Choi, J. Li, and N. Komuro, "A green and reliable communication modeling for industrial internet of things," Computers \& Electrical Engineering, 2016.

[33] A. Birk, B. Wiggerich, H. Bülow, M. Pfingsthorn, and S. Schwertfeger, "Safety, security, and rescue missions with an unmanned aerial vehicle (UAV): aerial mosaicking and autonomous flight at the 2009 european land robots trials (ELROB) and the 2010 response robot evaluation exercises (RREE)," Journal of Intelligent and Robotic Systems: Theory and Applications, vol. 64, no. 1, pp. 57-76, 2011.

[34] X. Fu, N. Zhang, A. Pingley, W. Yu, J. Wang, and W. Zhao, "The digital Marauder's map: a new threat to location privacy," in Proceedings of the 29th IEEE International Conference on Distributed Computing Systems Workshops, (ICDCS '09), pp. 589-596, IEEE, Montreal, Canada, June 2009.

[35] M. A. Goodrich, L. Lin, and B. S. Morse, "Using cameraequipped mini-UAVS to support collaborative wilderness search and rescue teams," in Proceedings of the International Conference on Collaboration Technologies and Systems (CTS '12), pp. 638-638, Denver, Colo, USA, May 2012.

[36] C. Liu, D. Fang, Z. Yang et al., "RSS distribution-based passive localization and its application in sensor networks," IEEE Transactions on Wireless Communications, vol. 15, no. 4, pp. 2883-2895, 2016.

[37] L. Shangguan, Z. Li, Z. Yang, and M. Li, “Otrack: towards order tracking for tags in mobile rfid systems," IEEE Transactions on Parallel \& Distributed Systems, vol. 25, no. 8, pp. 2114-2125, 2013.

[38] L. Shangguan and K. Jamieson, "The design and implementation of a mobile RFID tag sorting robot," in Proceedings of the 14th Annual International Conference on Mobile Systems, Applications, and Services (MobiSys '16), pp. 31-42, Singapore, June 2016.

[39] D. Gusenbauer, C. Isert, and J. Krosche, "Self-contained indoor positioning on off-the-shelf mobile devices," in Proceedings of the International Conference on Indoor Positioning and Indoor Navigation (IPIN '10), September 2010.

[40] J. Xu, W. Liu, F. Lang, Y. Zhang, and C. Wang, "Distance measurement model based on RSSI in WSN," Wireless Sensor Network, vol. 2, no. 8, pp. 606-611, 2010.

[41] Wikipedia, "Wardriving and warwalking [eb/ol]," 2013 https:// en.wikipedia.org/wiki/Wardriving.

[42] J. Scherer, S. Yahyanejad, S. Hayat et al., "An autonomous multiUAV system for search and rescue," in Proceedings of the 1st ACM Workshop on Micro Aerial Vehicle Networks, Systems, and Applications for Civilian Use (DroNet '15), pp. 33-38, Florence, Italy, May 2015.

[43] C. Han, K. Wu, Y. Wang, and L. M. Ni, "WiFall: device-free fall detection by wireless networks," in Proceedings of the 33rd IEEE Conference on Computer Communications (IEEE INFOCOM '14), pp. 271-279, Toronto, Canada, May 2014.

[44] L. Shangguan, Z. Yang, A. X. Liu, Z. Zhou, and Y. Liu, "Relative localization of RFID tags using spatial-temporal phase profiling," in Proceedings of the 12th USENIX Symposium on Networked Systems Design and Implementation (NSDI '15), pp. 251-263, USENIX, Oakland, Calif, USA, May 2015.

[45] A. Karimoddini, H. Lin, B. M. Chen, and T. Heng Lee, "Hybrid formation control of the unmanned aerial vehicles," Mechatronics, vol. 21, no. 5, pp. 886-898, 2011. 

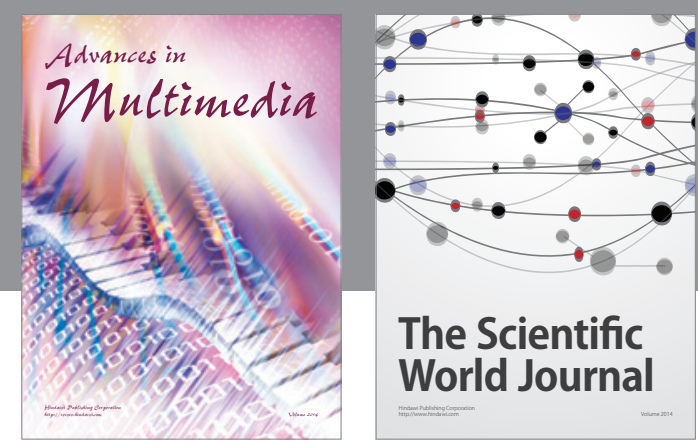

The Scientific World Journal
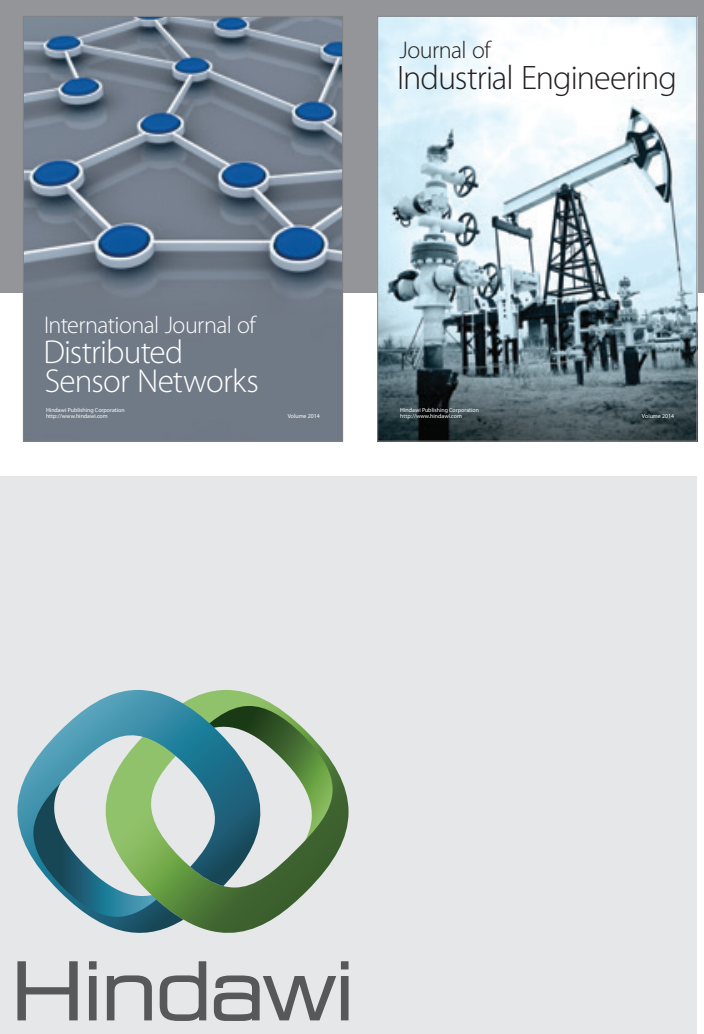

Submit your manuscripts at

https://www.hindawi.com

\section{Computer Networks} and Communications
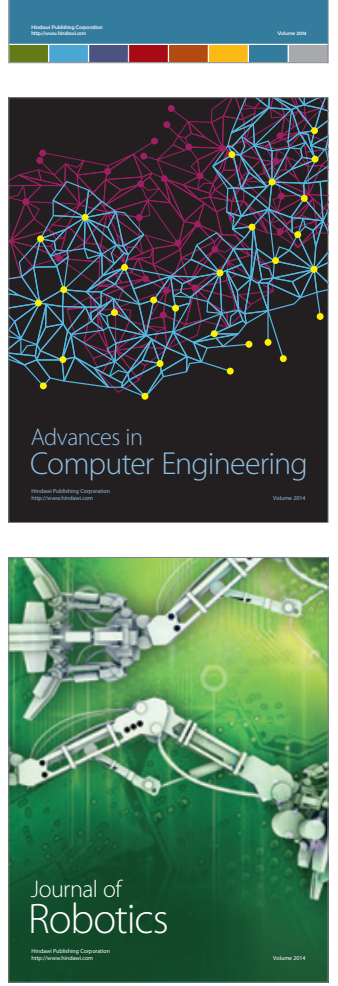
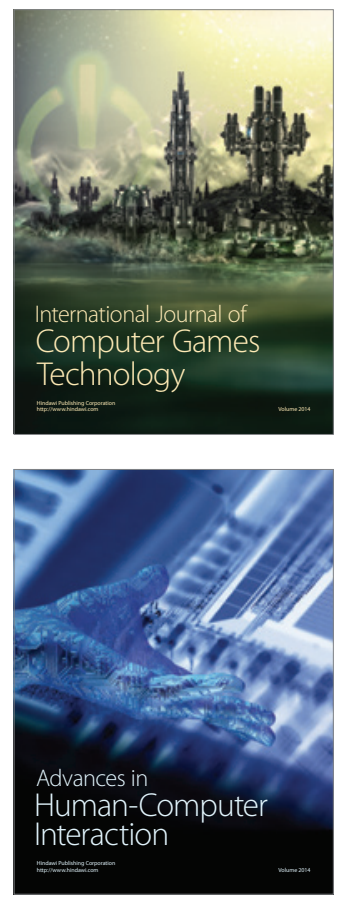
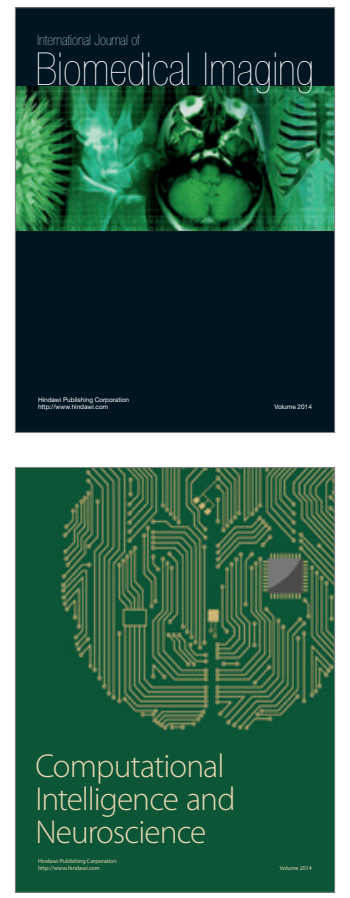
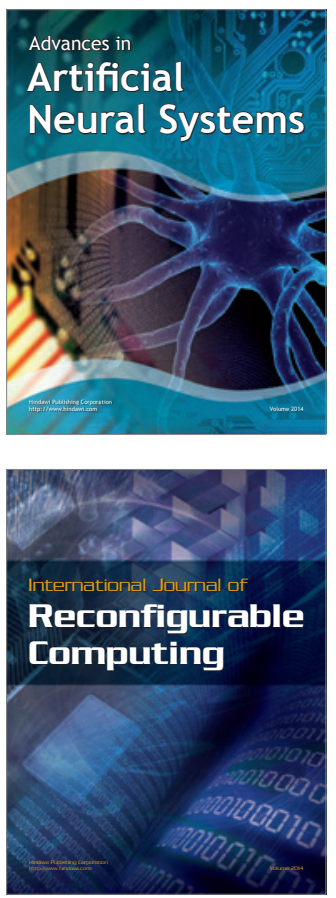
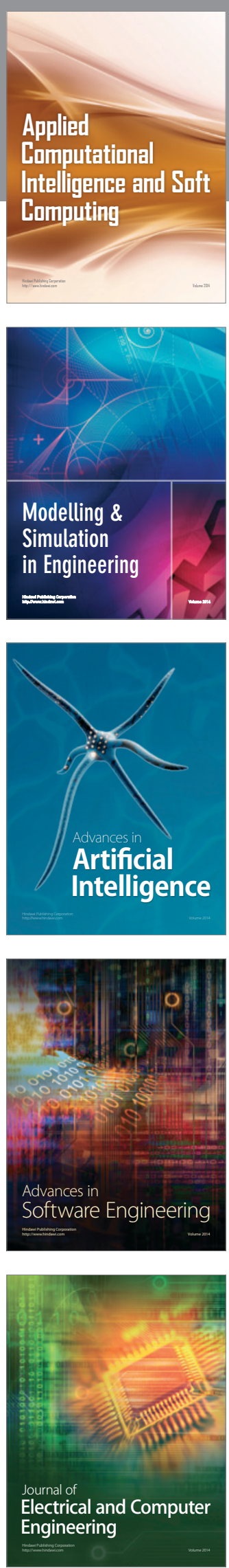